\title{
Deltopectoral flap reconstruction of the posterior pharyngeal wall - a simple and functional solution to the reconstruction of circumferential laryngopharyngeal defects
}

\author{
Swee Kang ${ }^{1}$, Sabih Qamar ${ }^{1}$, Theofano Tikka ${ }^{1}$, and Thomas Milner ${ }^{1}$ \\ ${ }^{1}$ Monklands Hospital
}

March 29, 2021

\begin{abstract}
Objectives To review the operative feasibility and functional outcomes following dual flap reconstruction of circumferential pharyngeal defects. Design Retrospective case series Setting University Hospital Monklands, a district general hospital Participants Eight patients undergoing either primary $(n=5)$ or salvage $(n=3)$ circumferential laryngopharyngectomy $+/$ - cervical oesophagectomy, followed by dual flap reconstruction, with a deltopectoral flap to reconstruct the posterior wall. Main Outcome Measures Operative complications, hospital stay and functional outcomes (speech and swallowing) Results The operation was feasible in all patients, with dual flap reconstruction using a deltopectoral flap, combined with a pectoralis major flap ( $\mathrm{n}=5$ ) or a supraclavicular flap $(n=3)$. All patients developed a small, lateralised, self-healing fistula at the site of the deltopectoral flap 3 -point junction. This did not require any intervention, or impact on adjuvant treatment. Functional outcomes were favourable, with all patients achieving oral diet. One patient required gastrostomy diet supplementation, and one patient required stricture dilatation. Of the patients able to receive a speech valve $(n=4)$, all achieved intelligible speech. Two patients could not receive a speech valve due to the inferior extent of the tumour resection, and trachea-oesophageal puncture has been delayed in 2 patients due to the COVID-19 pandemic. Conclusions Dual flap reconstruction of circumferential pharyngeal defects represents a simple, effective option for a complex reconstructive problem. The predictable operative recovery and favourable functional outcomes indicate that the use of both a deltopectoral flap and a second flap is a robust reconstructive solution.
\end{abstract}

\section{Abstract}

Objectives

To review the operative feasibility and functional outcomes following dual flap reconstruction of circumferential pharyngeal defects.

Design

Retrospective case series

Setting

University Hospital Monklands, a district general hospital

Participants

Eight patients undergoing either primary $(\mathrm{n}=5)$ or salvage $(\mathrm{n}=3)$ circumferential laryngopharyngectomy $+/$ cervical oesophagectomy, followed by dual flap reconstruction, with a deltopectoral flap to reconstruct the posterior wall.

Main Outcome Measures 
Operative complications, hospital stay and functional outcomes (speech and swallowing)

Results

The operation was feasible in all patients, with dual flap reconstruction using a deltopectoral flap, combined with a pectoralis major flap $(n=5)$ or a supraclavicular flap $(n=3)$. All patients developed a small, lateralised, self-healing fistula at the site of the deltopectoral flap 3-point junction. This did not require any intervention, or impact on adjuvant treatment. Functional outcomes were favourable, with all patients achieving oral diet. One patient required gastrostomy diet supplementation, and one patient required stricture dilatation. Of the patients able to receive a speech valve $(n=4)$, all achieved intelligible speech. Two patients could not receive a speech valve due to the inferior extent of the tumour resection, and trachea-oesophageal puncture has been delayed in 2 patients due to the COVID-19 pandemic.

\section{Conclusions}

Dual flap reconstruction of circumferential pharyngeal defects represents a simple, effective option for a complex reconstructive problem. The predictable operative recovery and favourable functional outcomes indicate that the use of both a deltopectoral flap and a second flap is a robust reconstructive solution.

Keypoints

- Circumferential pharyngeal defects represent a complex reconstructive problem

- Dual flap reconstruction using a deltopectoral flap and a second flap (a pectoralis major or a supraclavicular flap) offers a potential solution

- All patients developed a small, lateralised, self-healing fistula that required no intervention, and did not significantly impact on patient recovery

- Swallowing outcomes were favourable with all patients achieving oral diet, and only one patient requiring gastrostomy supplementation

- All patients who received a speech valve attained intelligible speech

Keywords: Pedicled flap; Pharyngectomy; Laryngectomy; Case series

Introduction

Circumferential laryngopharyngectomy and cervical oesophagectomy present a unique reconstructive challenge due to the complete loss of both the anterior and posterior pharyngeal walls, requiring mobilisation of adequate tissue to create the neopharynx. ${ }^{1-2}$ The radial forearm free flap (RFFF), the latissimus dorsi flap, the anterolateral thigh flap, the free jejunal flap (FJF) and the pectoralis major myocutaneous flap (PMMC) are more commonly described in the literature for reconstruction used either tubed or by suturing the flap in a 'horseshoe-shaped' fashion to the prevertebral fascia ${ }^{3-5}$ In the early surgical series, the use of free flaps was associated with a higher rate of post-operative fistula formation (up to 67\%) compared to PMMC flap reconstruction $(22 \%)^{6-7}$ but the percentage of such complications in the former groups has significantly improved in more recent studies (11-14\% fistula;14-16\% stenosis). ${ }^{8}$ However, the biggest challenge is regarding the long-term functional outcomes of swallow and speech, which remain poor and difficult to produce good outcomes consistently.

We describe our experience with the use of a deltopectoral flap for reconstruction of the posterior pharyngeal wall and another flap (pectoralis major or supraclavicular flap in our case series) to complete the reconstruction of the circumferential pharyngo-oesophageal defects.

\section{Methods}

\section{Patient Population}

A retrospective case series review was conducted in University Hospital Monklands, a district general hospital, from January 2005 (when the senior author SKK started his consultancy post), until December 2020. This identified all patients who had undergone circumferential laryngopharyngectomy $+/$ - cervical oesophagectomy with dual flap reconstruction using the deltopectoral (DP) flap to reconstruct the posterior wall and 
another flap to cover the defect anterolaterally. Eight patients were identified, with procedures performed between April 2018 and December 2020.

\section{Ethical considerations}

Institutional approval was attained following discussion at the local Head and Neck Oncology multidisciplinary team meeting and Caldicott Guardian approval was granted. This case series has been reported in line with the PROCESS guideline ${ }^{9}$.

\section{Surgical Technique}

Reconstruction is performed following laryngectomy and circumferential pharyngectomy $+/$ - cervical oesophagectomy. A DP flap is raised in the subfascial plane. The blood supply from the $2^{\text {nd }}$ and $3^{\text {rd }}$ internal mammary perforator arteries allows harvesting of a broad tissue flap with a width extending from the clavicle to the $5^{\text {th }}$ intercostal space, and a length reliably extending towards the shoulder tip. The flap is sutured onto the posterior oropharyngeal mucosa superiorly, and the proximal oesophagus inferiorly (Figure 1). In one individual, the superior extent of the flap repair extended to the level of the soft palate. Total time required to raise the DP flap and suture it to the inferior edge of the oropharynx is approximately 30 minutes.

A second flap is then harvested. This can be a free flap or a pedicled flap. In our series, a pectoralis major myocutaneous flap or a supraclavicular fasciocutaneous flap on the opposite side was used. This is to form the anterior and lateral pharyngeal wall. This second flap is raised and inserted in a similar manner to a partial pharyngeal patch repair. ${ }^{3,7}$

The distal part of the deltopectoral cutaneous tissue is left to form the posterior pharyngeal wall and skin de-epithelialisation is performed to a small middle segment of the flap, so that the only cutaneous tissue buried within the neck is the neopharynx itself (Figure 2). The skin of the deltopectoral flap near the stump of the oesophagus is sutured, thereby ensuring the integrity of the neo-posterior pharyngeal wall from oropharynx to oesophagus.

The second flap can then be sutured to the anterior mucosa of the proximal oesophagus, the DP flap laterally, and the tongue base superiorly, creating a conical neopharynx. Flap harvest sites are then closed primarily. A salivary bypass tube is positioned in the reconstructed neopharynx to help the healing process splinting it open, which is subsequently removed 3 weeks later, prior to commencement of oral diet. Six-month post-operative outcome is displayed in Figure 3.

\section{Results}

The case series includes 8 patients (Male:Female $=4: 4$ with a mean age of 68.6 years (range 55-82). Five procedures were performed as primary circumferential laryngopharyngectomies, and three patients underwent salvage operations following previous radical chemoradiotherapy (7 years, 9 years and 28 years prior to surgery). Primary tumour maximal diameters ranged from 17-75 mm (mean: $41.6 \mathrm{~mm}$ ).

The anterior pharyngeal wall was reconstructed with a pectoralis major flap for 5 patients and a supraclavicular flap for the remaining 3 patients. All patients developed a small pharyngocutaneous fistula, laterally in the neck, over the 3-point junction of the DP flap. In all patients this required no intervention, did not result in wound dehiscence, and closed spontaneously within 4-28 days (mean: 15.6). For the three patients requiring adjuvant (chemo)radiotherapy, there was no delay in treatment delivery.

Functional outcomes were favourable in the majority of patients. All patients have achieved normal $(\mathrm{n}=2)$ or soft $(n=6)$ diet, although one individual continues to require PEG tube dietary supplementation. Of the 6 patients that manage soft diet, one developed a low neopharyngeal stricture requiring repeated dilatations. A speech valve has been inserted in 4 patients, with all achieving intelligible speech, 2 patients are awaiting tracheo-oeosophageal puncture which has been postponed during the COVID-19 pandemic. Speech valve insertion was not possible for 2 patients, as the oesophagectomy level is significantly below the level of the stoma. Two patients died during follow up, one following tumour recurrence, and one unrelated to their malignancy. All results are summarised in table 1. 


\section{Discussion}

This case series presents an alternative method for reconstruction of circumferential pharyngeal defects using a dual flap technique, with the rotated deltopectoral flap as the posterior wall of the neopharynx. The purpose of this technique is to reconstruct the neopharynx with minimal soft tissue bulk in the limited central compartment space, and to create a wider, conical conduit for food passage. A single tubed flap, with the exception of the jejunal free flap, incorporates all the flap bulk in the central compartment, limiting the calibre of the neopharynx. The deltopectoral flap positions naturally on the prevertebral fascia. The second flap being used as a patch flap as opposed to a tubed flap, allows the majority of the flap bulk to be located in the lateral neck.

To maintain continuity of the neopharynx, the flaps need to be sutured stepwise to the posterior pharyngeal wall postero-superiorly and to the tongue base supero-anteriorly. Similarly, the flaps are sutured inferiorly to the cervical oesophageal remnant forming a continuous food passage. This resulted in a conical shaped neopharynx (Figure 2), which resembles the natural pharynx more than a tubed flap reconstruction. The authors theorise that this wider, conical neopharynx would both enhance the swallow function, and allow improved air passage for speech. Our limited series seems to support this, with good functional outcomes reported.

The additional advantage to the DP flap, is that it can be rapidly raised and positioned on the posterior wall, requiring minimal de-epithelialisation. This also converts a complex circumferential defect into a 'partial' defect, and consequently, does not contribute significantly to the surgical time.

To the authors knowledge, the combination of a DP and a second flap has never been described before in the literature. Various techniques have been described for reconstructing circumferential defects, including tubed free flaps, tubed pedicled flaps, and suturing of flaps onto the prevertebral fascia. A recent multicentre study concluded that the type of flap used during reconstruction does not impact on the post-operative swallowing outcomes with just over half of patients being able to gain normal diet post-operatively ( $54 \%$ with any flaps; $63 \%$ RFFF. 53\% PMMC, 58\% FJF) with no significant difference between the different flaps being used. 5 Nevertheless, other series have demonstrated superior functional outcomes when free flaps were used $(58 \%$ - 63\% normal feeding; $21-25 \%$ stricture requiring dilatation) compared to tubed PMMC or PMMC with the prevertebral fascia forming the posterior pharyngeal wall (18-40\% stenosis requiring dilatation; $53-91 \%$ adequate oral nutrition; $43 \%$ satisfactory vocal function). ${ }^{10-13}$

In our case series, all patients ultimately achieved oral diet intake, with 7 patients achieving sufficient intake to support their complete nutrition. Only one patient developed a stricture requiring repeated dilatation. All patients who were able to receive a tracheooesophageal puncture attained intelligible speech. The need for post-operative dilatation of the neopharynx is frequently reported in the literature when tubed free or single pedicle flaps are used for reconstruction of circumferential pharyngolaryngeal defects. Dilatation figures vary from $21 \%$ with the FFF to $40 \%$ with PMMC. ${ }^{5}$

The main weakness of the dual flap technique lies in the likelihood of patients developing a small fistula. This occurs at the de-epithelialised region on the DP flap. While an important consideration in the perioperative period, this has not impacted on subsequent care or adjuvant treatment delivery in any of the patients involved. The fistula has always remained small and low volume, requiring minimal/no intervention, and the authors are of the opinion that the long-term functional outcome advantages outweigh the consequences of fistula development. In fact, it may be that the small fistula is advantageous, allowing a means of controlled drainage, as none of the patients have developed significant tissue breakdown. The factors that pre-dispose to fistulae have been investigated extensively in previous studies, including comparing between free and pedicled tissue flaps. ${ }^{14-16}$ Despite a recent trend towards increasing free flap use, morbidity profiles appear to be similar between free and pedicled flaps ${ }^{16}$. Although all patients in our case series developed a fistula, the short-lived nature, and the rapid healing indicate that dual flap use appears to be a robust reconstruction technique.

Conclusion 
Dual DP/second flap reconstruction of circumferential pharyngeal defects represents a simple, effective solution to a complex reconstructive problem, with the DP flap converting the complex, circumferential defect into a 'partial' defect. Our series demonstrated the reliability of the techniques to provide reasonable quality of life to patients. The authors recommend this as an alternative to the single pedicled or free flap tubed reconstruction for large circumferential pharyngeal defects.

\section{References}

1. Lewin JS, Barringer DA, May AH, Gillenwater AM, Arnold KA, Roberts DBet al. Functional outcomes after circumferential pharyngoesophageal reconstruction. Laryngoscope.2005;115(7):1266-71

2. Anthony JP, Singer MI, Mathes SJ. Pharyngoesophageal Reconstruction Using the Tubed Free Radial Forearm Flap. Clin Plast Surg.1994;21(1):137-47.

3. Spriano, G. Piantanida, R., Pellini, R. Hypopharyngeal reconstruction using pectoralis major myocutaneous flap and pre-vertebral fascia.Laryngoscope . 2001;111(3): 544-547

4. Jegoux F, Ferron C, Malard O, Espitalier F, Beauvillain de montreuil C. Reconstruction of Circumferential Pharyngolaryngectomy Using a 'Horseshoe-Shaped' Pectoralis Major Myocutaneous Flap. $J$ Laryngol Otol . 2007;121(5):483-8.

5. Morinière S, Gorphe P, Espitalier F, Blanchard D, Fakhry N, Saroul Net al. Assessment of swallowing function after circumferential pharyngolaryngectomy. A multicenter study by the GETTEC group.Eur Ann Otorhinolaryngol Head Neck Dis . 2019;136(1):3-5.

6. Kelly KE, Anthony JP, Singer M. Pharyngoesophageal Reconstruction Using the Radial Forearm Fasciocutaneous Free Flap: Preliminary Results.Otolaryngol Head Neck Surg. 1994;111(1):16-24.

7. Spriano, G., Pellini, R., Roselli, R. Pectoralis major myocutaneous flap for hypopharyngeal reconstruction. Plastic and Reconstructive Surgery . 2002;110 (6):1408-1413

8. Reiter M, Baumeister P. Reconstruction of Laryngopharyngectomy Defects: Comparison Between the Supraclavicular Artery Island Flap, the Radial Forearm Flap, and the Anterolateral Thigh Flap. Microsurgery 2019;39(4):310-315.

9. Mura F, Bertino G, Occhini A, Mevio N, Scelsi D, Benazzo M. Advanced Carcinoma of the Hypopharynx: Functional Results After Circumferential Pharyngolaryngectomy With Flap Reconstruction. Acta Otorhinolaryngol Ital. 2012;32(3):154-7.

10. Agha RA, Sohrabi C, Matthew G, Franchi T, Kerwan A, O'Neill N for the PROCESS group. The PROCESS 2020 guideline: updating consensus preferred reporting of case series in surgery (PROCESS) guidelines.Int J Surg . 2020; 84: 231-5

11. Espitalier F, Ferron C, Leux C, Jégoux F, Durand N, Beauvillain de Montreuil C et al. Results After U-shaped Pectoralis Major Myocutaneous Flap Reconstruction of Circumferential Pharyngeal Defects. Laryngoscope . 2012;122(12):2677-82

12. Burke MS, Kaplan SE, Kaplowitz LJ, Lotempio MM, Hicks WL Jr, Rigual NRet al. Pectoralis major myocutaneous flap for reconstruction of circumferential pharyngeal defects. Ann Plast Surg.2013;71(6):649-51.

13. Chan YW, Ng RW, Liu LH, Chung HP, Wei WI. Reconstruction of circumferential pharyngeal defects after tumour resection: reference or preference. J Plast Reconstr Aesthet Surg. 2011;64(8):1022-1028.

14. Piazza C, Taglietti V, Nicolai P. Reconstructive options after total laryngectomy with subtotal or circumferential hypopharyngectomy and cervical oesophagectomy. Curr Opin Otolaryngol Head Neck Surg . 2012; 20(2): 77-88

15. Sun AH, Xu X, Sasaki CT, Ariyan S, Steinbacher DM. A thirty-year experience with head and neck flap reconstruction. J Craniofac Surg . 2017; 28(5): 1354-61.

16. Sayles M, Grant DG. Preventing pharyngo-cutaneous fistula in total laryngectomy: a systematic review and meta-analysis.Laryngoscope . 2014; 124(5): 1150-63 


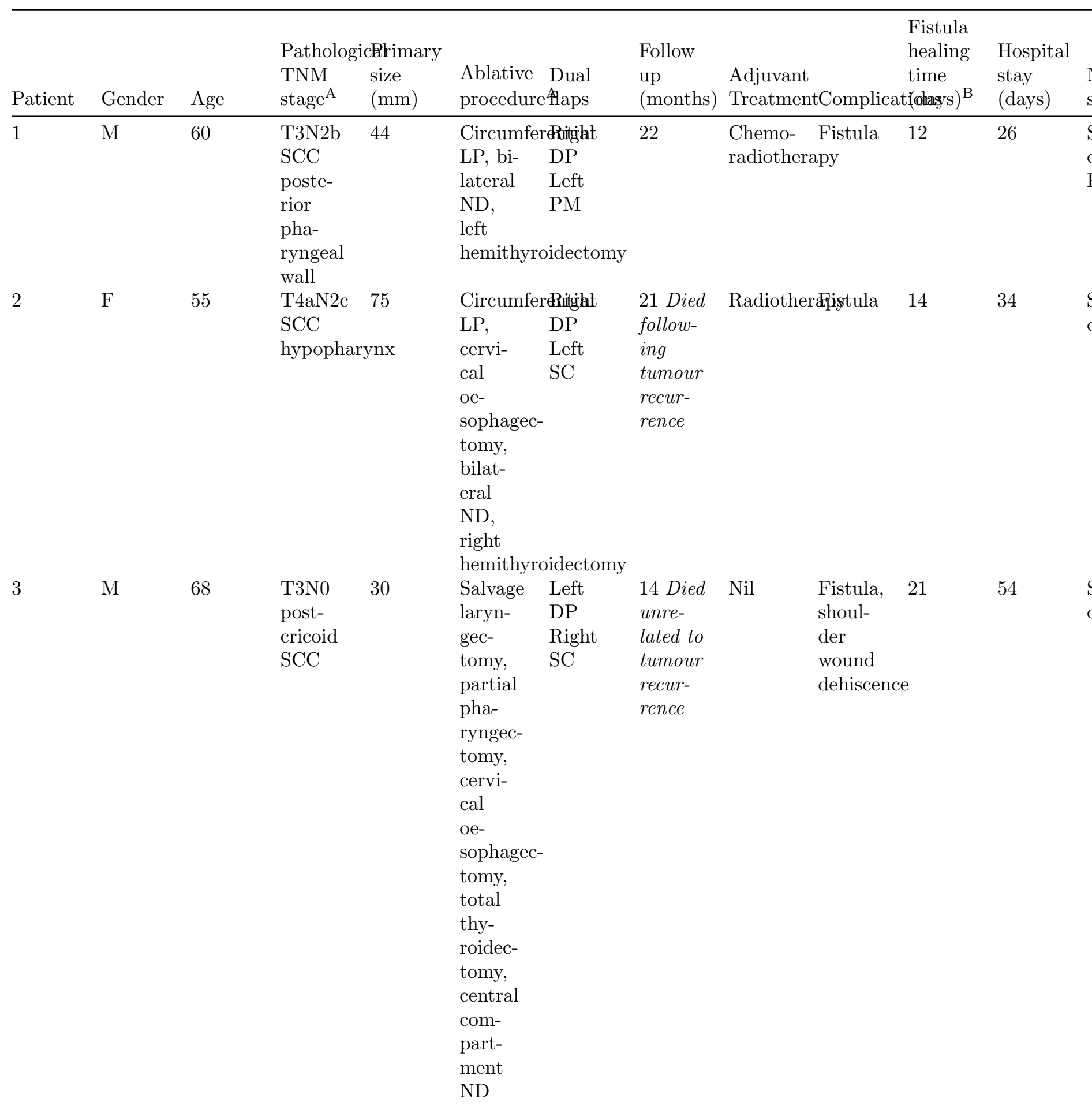




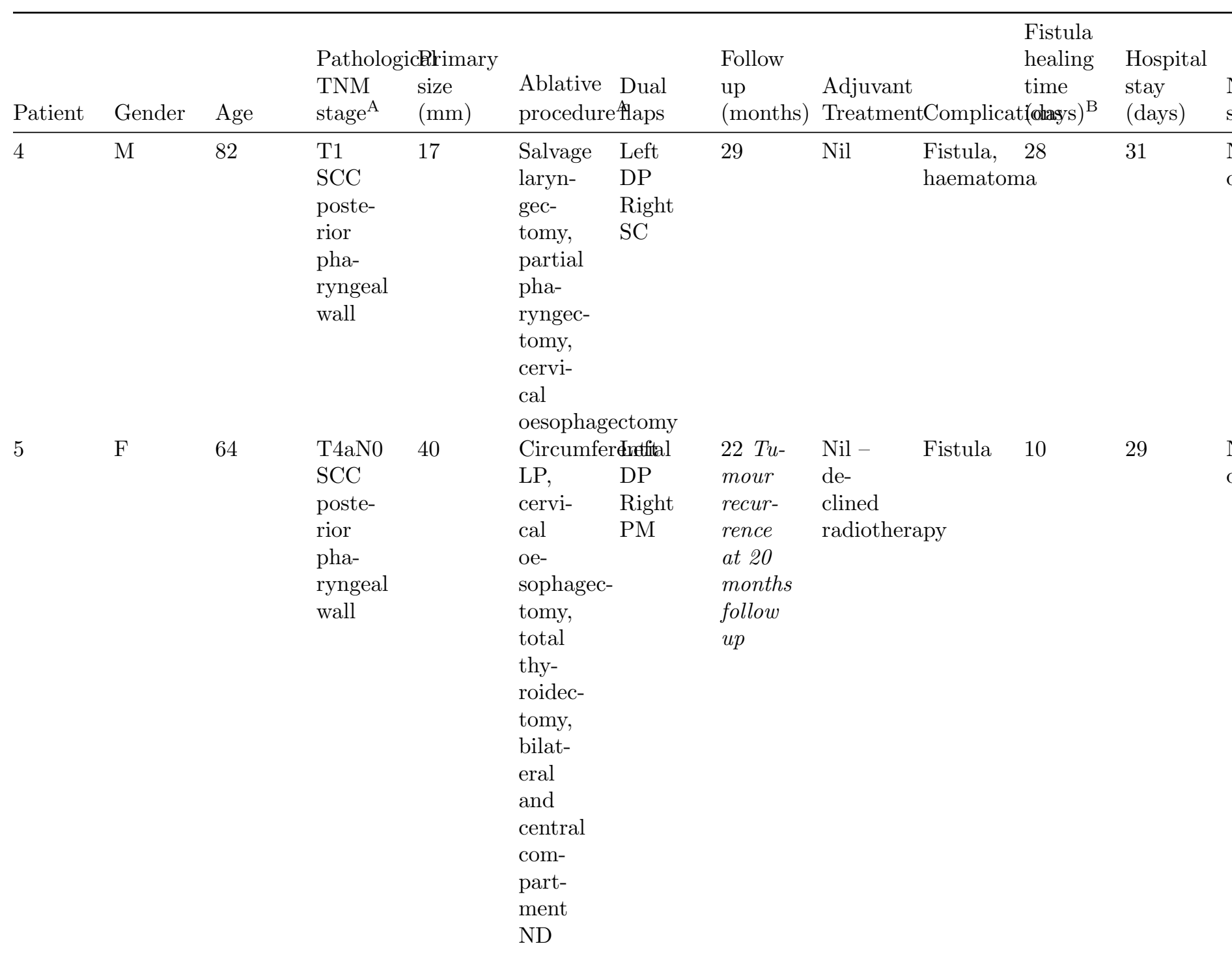




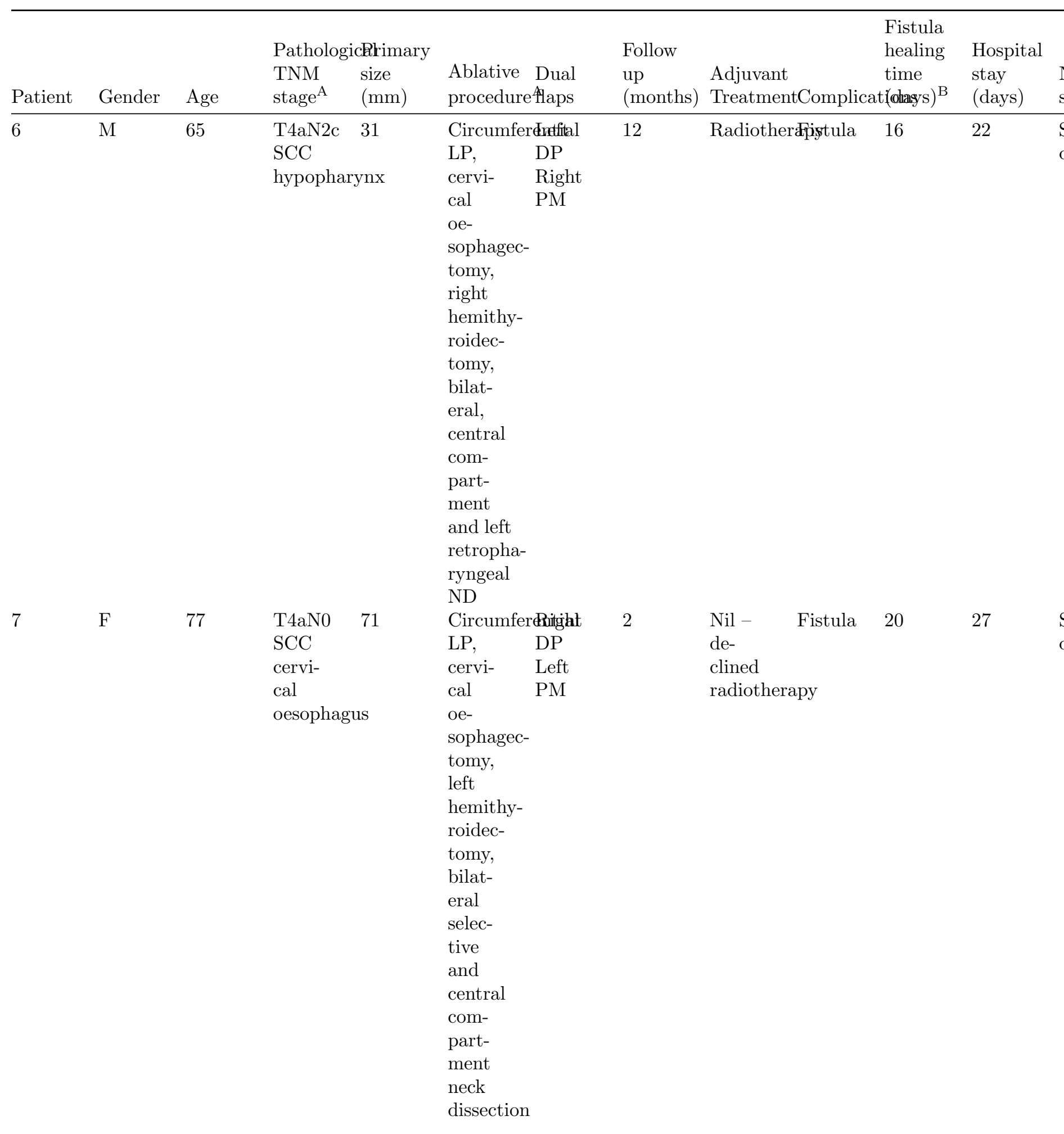




\begin{tabular}{|c|c|c|c|c|c|c|c|c|c|}
\hline Patient & Gender & Age & $\begin{array}{l}\text { Patholog } \\
\text { TNM } \\
\text { stage }^{A}\end{array}$ & $\begin{array}{l}\text { size } \\
(\mathrm{mm})\end{array}$ & $\begin{array}{l}\text { Ablative Dual } \\
\text { procedureflaps }\end{array}$ & $\begin{array}{l}\text { Follow } \\
\text { up } \\
\text { (months) }\end{array}$ & $\begin{array}{l}\text { Adjuvant } \\
\text { TreatmentComplica }\end{array}$ & $\begin{array}{l}\text { Fistula } \\
\text { healing } \\
\text { time } \\
\text { t(dosys) }^{\mathrm{B}}\end{array}$ & $\begin{array}{l}\text { Hospital } \\
\text { stay } \\
\text { (days) }\end{array}$ \\
\hline 8 & $\mathrm{~F}$ & 78 & $\begin{array}{l}\text { T2 } \\
\text { post- } \\
\text { cricoid } \\
\text { SCC }\end{array}$ & 25 & $\begin{array}{ll}\text { Salvage } & \text { Left } \\
\text { laryn- } & \text { DP } \\
\text { gec- } & \text { Right } \\
\text { tomy, } & \text { PM } \\
\text { partial } & \\
\text { pha- } & \\
\text { ryngec- } & \\
\text { tomy, } & \\
\text { cervi- } & \\
\text { cal } & \\
\text { oesophagectomy }\end{array}$ & 2 & Fistula & 4 & 18 \\
\hline
\end{tabular}

Table 1

Table displaying individual patient demographics and clinical information

A $\mathrm{SCC}=$ squamous cell carcinoma, $\mathrm{LP}=$ laryngopharyngectomy, $\mathrm{ND}=$ neck dissection (selective/modified radical)

B All patients developed a small, low volume fistula at the three-point junction at the site of the desquamated DP flap

Figure Legends

Figure 1: DP flap raised and sutured into position proximally and distally. Note the conical shape of the neopharynx generated naturally upon positioning the DP flap. A = Region of DP flap forming neopharynx. $\mathrm{B}=$ Region of DP flap to be de-epithelialise.

Figure 2: Neopharynx being created from the DP flap (A) and SC flap (B) around a salivary bypass tube. Laryngeal stoma is notable inferiorly. $\mathrm{C}=$ De-epithelialised $\mathrm{SC}$ flap. $\mathrm{D}=$ De-epithelialised DP flap. $\mathrm{E}=$ Pharyngeal remnant

Figure 3: Post-operative outcome - an external view of the neck and stoma. A = Deltopectoral flap 

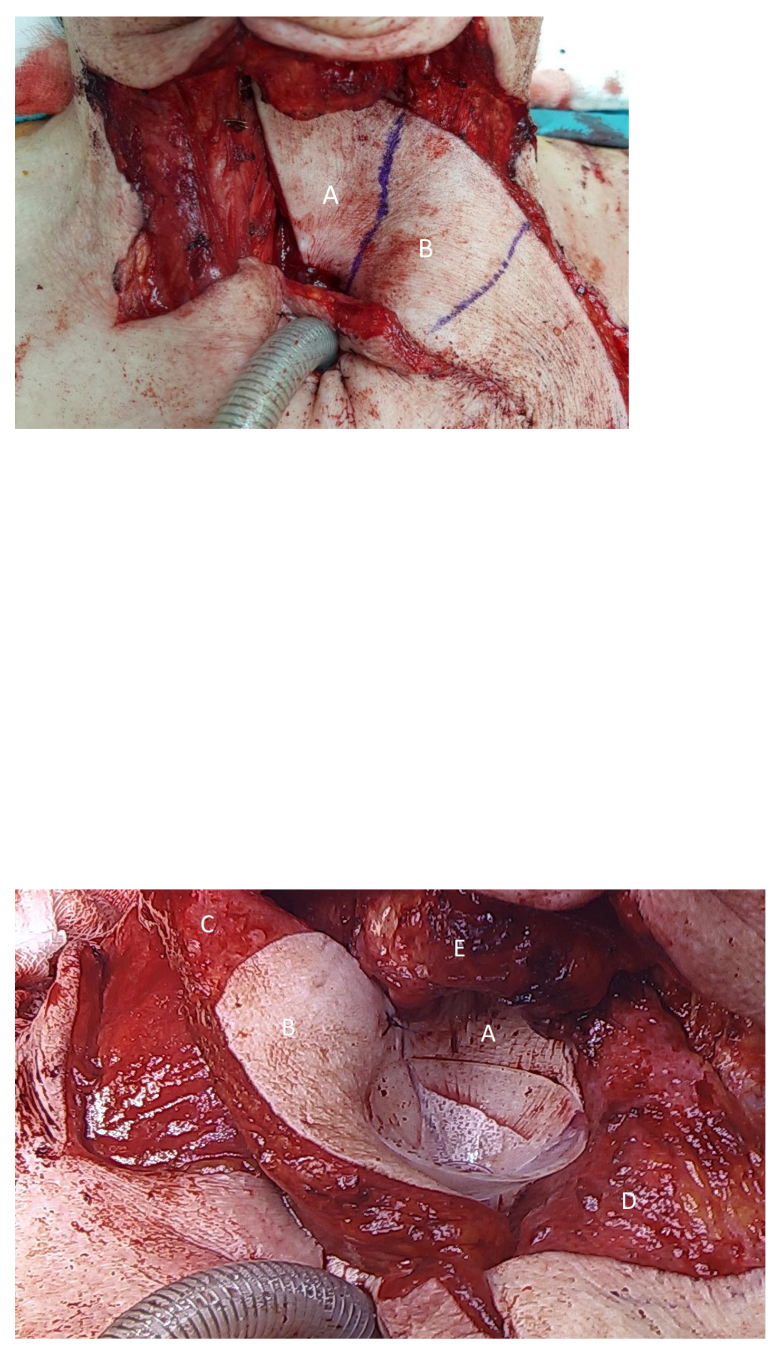


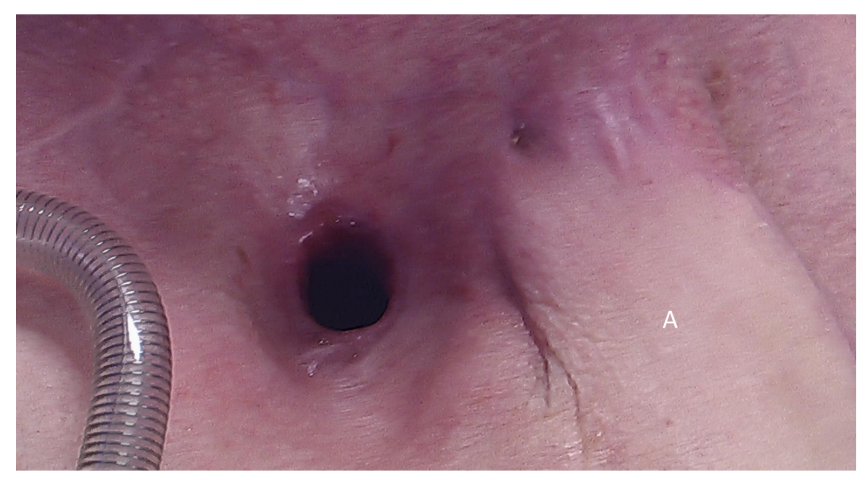

